\title{
2-Sübstitüeoksazolo (4,5-b) Piridin Türevlerinin Sentez ve Yapı Açıklamaları
}

Synthesis and Structure Elucidations of 2-Substitutedoxazolo (4,5-b) Pyridine Derivatives

Seçkin ÖZDEN* İsmail YALÇIN* Tuncel ÖZDEN*

\section{ÖZET}

Bu çalışmada, 2. konumda, homolog bir dizi içinde düz ve dallanmış alkil grupları ile bunların halkalı karşıtlarını içeren oksazolo(4,5-b) piridin türevleri sentez edilmiştir. Bu bileşiklerin sentezleri 2-amino-3-hidroksipiridinin, ilgili karboksilik asitler veya asit anhidritleri ile polifosforik asit veya polifosforik asit-fosfor pentaoksit varl1ğında ısıtılması ile gerçekleştirilmiştir.

Bu şekilde hazırlanan 12 türevden, 9 tanesi ilk kez bu çalışmada sentez edilmiştir.

Elde edilen türevlerin saflıkları, İT.K. ile kontrol edildikten sonra, ergime veya kaynama noktaları saptanmış ve yapıları UV, IR, NMR ve Kütle Spektrumları ile açıklanmıştır.

\section{SUMMARY}

In this research, 2-n-alkly, branched alkyl and cycloalkyl substituted oxazolo (4,5-b) pyridine derivatives were synthesized. 12 compouds had been prepared and 9 of them were original.

These compounds were synthesized by consensing 2-amino-3hydroxypyridine with carboxylic acids or acid anhydrides in the pre-

* Farmasötik Kimya Anabilim Dalı, Eczacılık Fakültesi, Ankara Üniversitesi 
sence of polyphosphoric acid or polyphosphoric acid-phosphor pentaoxide mixture. The purity and chemical structures had been elucidated by TLC, melting or boiling points and instrumental analysis.

Anahtar Kelimeler: Oksazolo (4,5-b) piridin, Polifosforik asit-fosfor pentaoksit, 2-Amino-3-hidroksipiridin, UV, IR, NMR, K ÜT L E S P E K T R U M LAR I

İlk kez 1957 yılında FRASER ve TIT T EN S R tarafından sentezlenen oksazolo (4,5-b) piridin türevleri üzerinde son yirmi yıl içinde yoğun çalışmalar yapılmaktadır (1). Özellikle SHEN ve Arkadaşları 1972 yılından bu yana yaptıkları çalışmalarda 2. konumda heterosiklik halkalar ve sübstitüefenil grupları taşıyan bir dizi oksazolopiridin türevlerini sentezlemişlerdir (2-6). Bu türevlerde bulunan antienflamatuvar, analjezik ve antipiretik etkiler bazik özellikteki nonsteroid antienflamatuvar ilaçlara kaynak teşkil etmektedir (2). Bu etkilerin mekanizması henüz kesin olarak açıklanmış değildir. Ancak histamin, serotonin ve prostaglandinler gibi bazı endojen maddelerin enflamasyon ve ağrı mediyatörleri olduğu bilinmektedir.

$\mathrm{Bu}$ görüşten hareketle yapılan çalışmada histamin reseptörleri üzerine etki gösterebileceği düşünülen 2-sübstitüeoksazolo (4,5-b)piridin türevleri hazırlanmıştır. 2. konumdan gerçekleştirilen sübstitüsyonlar sonucunda, düz zincirli alkil grupları ve bunların dallanmış şekilleri ile, ayrıca moleküldeki uzaysal değişikliklerin ne ölçüde etkin olduğunu anlıyabilmek amaciyla düz zincirli gruplarla aynı sayıda karbon atomu içeren doymuş halkalı karşıtları sentez edilmiştir. Böylece türevlerin homolog bir dizi içinde sentezlerinin gerçekleştirilmesi düşünülmüştür.

Sentezi yapılan maddelerin biyolojik etkilerinin saptanması ve yapı-etki ilişkilerinin incelenmesi ayrı bir çalışma olarak sunulacaktır.

\section{DENEL KISIM}

\section{Materyal:}

Çalışmalarda kullanılan çözücüler (Merck, Aldrich, Riedel) teknik veya analitik niteliktedir. 2-amino-3-hidroksipiridin (Aldrich) karboksilik asitler, anhidritler, polifosforik asit ve fosfor pentaoksit (Fluka, Merck) arı bileşikleridir. 
Aletsel analiz çalışmalarında, spektral saflıkta potasyum bromür (Merck), kloroform-d (Merck), kromatografi çalışmalarında adsorban olarak kiselgel HF254 (Camag) kullanılmıştır.

Elektronik Cihazlar: Ultraviyole Spektrofotometresi; Pye Unicam SP 1700, İnfrared Spektrofotometresi; Pye Unicam SP 1025, Nükleer Manyetik Rezonans Spektrometresi; Perkin Elmer R32, Kütle Spektrometresi; Dupont 21 490-B

Bileşiklerin ergime noktaları, Mettler FP-5 ve FP-51, kaynama noktaları ise, Büchi SMP-20 cihazları ile saptanmıştır.

\section{Metod:}

Oksazolopiridilerin Elde Ediliş Metodları: Oksazolo (4,5-b)piridin türevlerinin elde ediliş şekilleri çoğunlukla 2-amino-3-hidroksipiridinden hareketle yapılan halka kapatma reaksiyonlaridir (1,711). Başlangıç maddesi olarak 2-amino-3-hidroksipiridinin kullanılmadı̆̆ 1 bazı sentez çalışmaları da bulunmaktadır. $(1,7,12)$.

$\mathrm{Bu}$ halka sistemi üzerinde çalışan SHEN ve Ark. ise, 2-sübstitüeoksazolo (4,5-b) piridin türevlerinin eldesi için en uygun sentez yönteminin karboksilik asit veya karboksilik asit anhidritlerinin, 2amino-3-hidroksipiridin ile polifosforik asit veya polifosfat esteri varl1ğında isıtmak olduğunu belirtmektedirler (2-6). Uyguladikları sentez yöntemleri ve reaksiyon şartları Şekil 1 de verilmiştir.

Oksazolopiridin Halka Sisteminin Oluşturulması: Bu çalışmada hazırlanan bileşikler, 2-amino-3-hidroksipiridin (0,045 mol) ve asit anhidritinin $(0.135 \mathrm{~mol})$ polifosforik asit varlı̆̆ında veya anhidrit yerine karboksilik asit $(0.135 \mathrm{~mol})$ kullanıldı̆̆ında polifosforik asitfosfor pentaoksit karışımında ısıtılması ile elde edildi. Reaksiyon geri çeviren soğutucu altında, her bileşiğe göre değişen ısılarda 1 saat manyetik karıştırıcı üzerinde karıştırılarak gerçekleştirildi. Bu süre sonunda reaksiyon içeriği henüz sıcak iken buzlu suya döküldü ve ayrıca dıştan soğutulup karıştırılmaya devam edilirken \% 10 sodyum hidroksit çözeltisi ile ortam alkali yapıldı. Alkali çözelti benzenle tüketildi. Benzenli çözelti turnusol kağıdına nötr reaksiyon verene kadar su ile yıkandı ve susuz sodyum sülfatla suyundan kurtarıldı. Daha sonra vakumda kuruluğa kadar uçuruldu. Bu şekilde elde edilen kalıntı, eter 


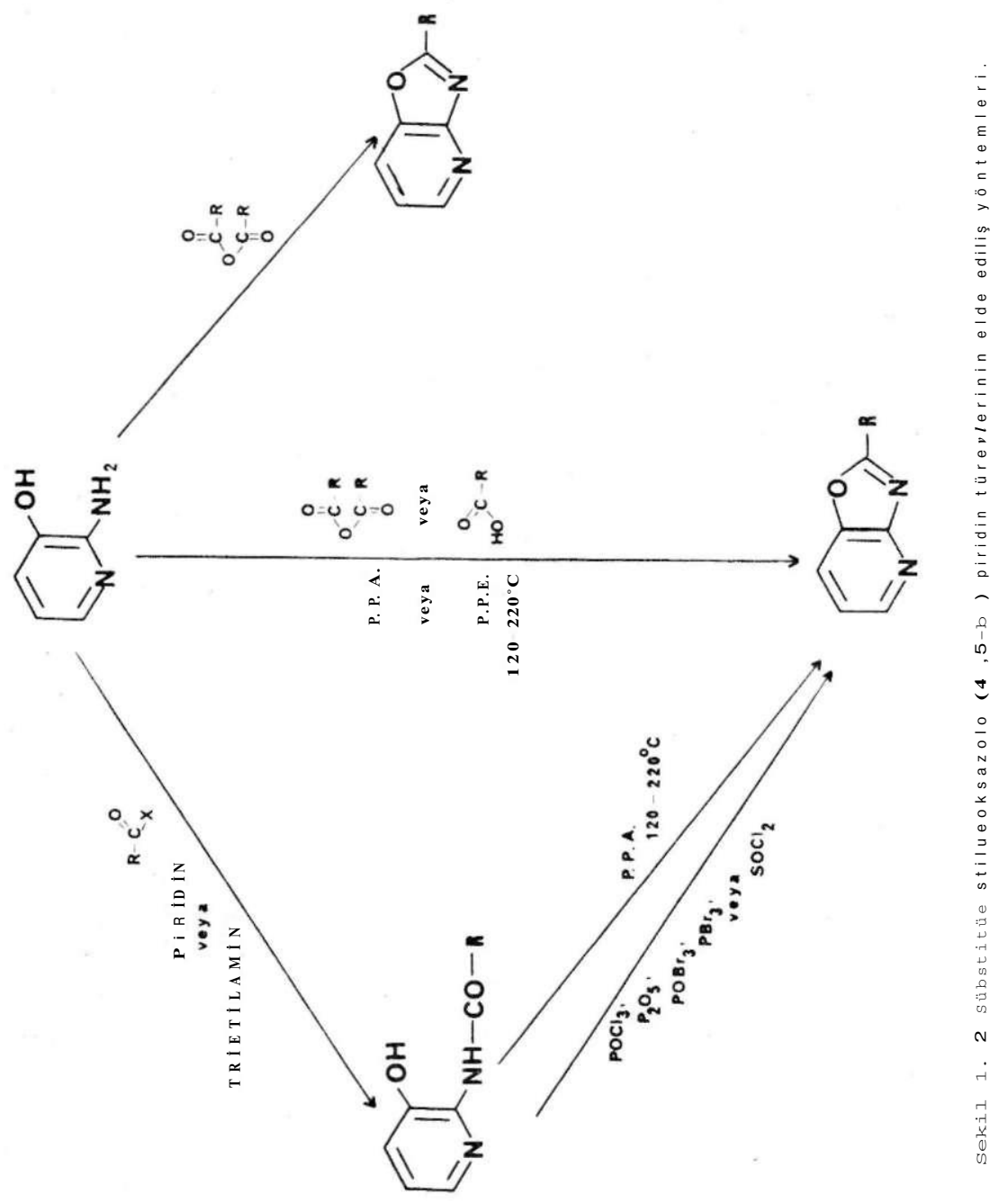


içinde çözüldü ve bu çözeltinin rengi aktif kömür ve alüminyum oksit yardımıla giderildi. Çözelti tekrar vakumda tamamen uçuruldu. Kalıntı sıvı ise ortamdan alındı. Böylece sıvı olan türevler izole edilmiş oldu. Kalıntı katı ise eterde çözüldü ve petrol eteri ile doyurularak soğukta bekletildi. Kristal halinde ayrılan bileşik süzülerek alındı. $30^{\circ} \mathrm{C}$ de vakum etüvünde kurutulan türevlerin hepsinin ince tabaka kromatografisi ile saflı̆̆ kontrol edildi.

İnce tabaka kromatografisinde kullanılan en uygun solvan sistemi kloroform/metanol (40:1) olup, adsorban ise kiselgel $\mathrm{HF}_{254}$ dür. $0.300 \mathrm{~mm}$ kalınlığında hazırlanan plaklar, $105{ }^{\circ} \mathrm{C}$ lik etüvde 1 saat tutularak aktive edildi. Lekelerin belirlenmesinde, ultraviyole 1şığından, Dragendorff reaktifi ve \% 10 luk demir -III- klorür çözeltisinden yararlanıldı.

\section{Sentez Edilen Oksazolopiridin Türevlerinin Yapı Açık- lamaları :}

Sentezlenen bileşiklerin, UV, IR, NMR ve Kütle spektrumları alınarak bu spektrumlar yardımı ile her bileşiğin yapısı açıklandı.

Ultraviyole Spektrumları: Sentez edilen tüm bileşiklerin dağılma katsayıları saptanırken, 1- oktanol içerisinde çözülerek türevlerin UV spektrumları alındı̆̆ından başka bir çözücü kullanılarak işlemin tekrarlanılmasına gerek duyulmamıştır. Tarama 190-400 nm ler arasında yapılmış, türevlerin benzer spektrum göstermekte ve aynı dalga boyunda maksimum verdikleri görülmüştür. Türevlerdeki değişiklik homolog bir dizi içinde bir karbon artış1 şeklinde olduğundan ve alkil grupları spektrumu normal olarak etkilemeyeceğinden her türev için benzer spektrum alınması doğaldır.

Bütün bileşiklerin UV spektrumlarında 226 ve $278 \mathrm{~nm}$ de birer maksimum ve $211 \mathrm{~nm}$ de de bir omuz görülmektedir.

IR Spektrumları: Bileşiklerin IR spektrumları potasyum bromür ile pellet haline getirilerek alınmıştır. IR spektrumlarında, aşağıda verilen bantlar tüm türevler için benzerdir:
1) $3120-3000 \mathrm{c} \mathrm{m}^{-1}$
$=\mathrm{C}-\mathrm{H}$ gerilimi
2) $2990-2880 \mathrm{~cm}^{-1}$
$-\mathrm{C}-\mathrm{H}$ gerilimi
3) $1622-1610 \mathrm{c} \mathrm{m}^{-1}$
$\mathrm{C}=\mathrm{N}$ gerilimi 

4) $1490-1480 \mathrm{c} \mathrm{m}^{-1}$
5) $1420-1415 \mathrm{~cm}^{-1}$
6) $1390-1370 \quad \mathrm{~cm}^{-1}$
7) $1265-1150 \quad \mathrm{~cm}^{-1}$
8) $810 \mathrm{~cm}^{-1}$

- C-H eğilimi

Oksazol halkası halka vibrasyonu - C-H gerilim

C-O-C gerilim

C-H plan dişı eğilimi (1, 2-disübstitüe aromatik halka)

Elde edilen türevlerin spektrumlarında, sentez başlangıç bileşiklerinin bulunabileceğim gösteren $\mathrm{N}-\mathrm{H}, \mathrm{O}-\mathrm{H}, \mathrm{COOH}$ gerilim bantları ile reaksiyonun amit basamağında kaldığını belirten, N-H, O-H, C-O gerilim bantlarına rastlanılmamıştır.

NMR Spektrumları: Dötore kloroform içinde çözülerek N M R spektrumları alınan türevlerde, oksazolopiridin halkası protonlarının sinyalleri hepsinde aynı alanda görülmektedir. 2. konumdaki sübstitüentlerin protonlarının sinyalleri ise gruplara bağlı olarak değişmektedir.

Kütle Spektrumları: Bütün spektrumlarda oksazolopiridin yapısı taşıyan bileşiklere özgü olan parçalanmalar görülmektedir. Bu ortak parçalanmalar: 1) 2. Konumda alkil bulunduran oksazol halkasına ait olan m/e 134 iyonu. 2) Oksazol halkasının yarılması sonucu oluşan m/e 93 iyonu. 3) Oksazol halkasının parçalanması sonucu oluşan m/e 93 iyonundan CO çıkışı ile oluşan m/e 65 iyonu.

2. konumda bulunan alkil gruplarının durumlarına göre parçalanmalar iki şekilde olmaktadır. Hem düz veya dallanmış, hem de halkalı alkil gruplarının parçalanması normal sırada olmaktadır. Şekil 2 de 2-n-hekziloksazolo (4,5-b) piridin ve 2-sikloheksiloksazolo (4,5-b) piridin türevlerinin alkil parçalanma şekilleri örneklenmiştir.

$\mathrm{Bu}$ çalışmada sentezi yapılan oksazolopiridin türevleri ile bunların ergime veya kaynama dereceleri Tablo 1 de gösterilmiştir.

\section{1) 2- Metiloksazolo (4,5-b) piridin (Bileşik No: 1)}

Genel yöntem uygulanan sentezde, $5 \mathrm{~g}$ (0.045 mol) 2-amino-3hidroksipiridin, $13.2 \mathrm{~g}(0.135 \mathrm{~mol})$ asetik asit anhidriti kullanıldı. $110-20{ }^{\circ} \mathrm{C}$ de yürütülen reaksiyon sonucunda $1.18 \mathrm{~g}$ kristal bileşik elde edildi. (Verim: \% 19.37) 

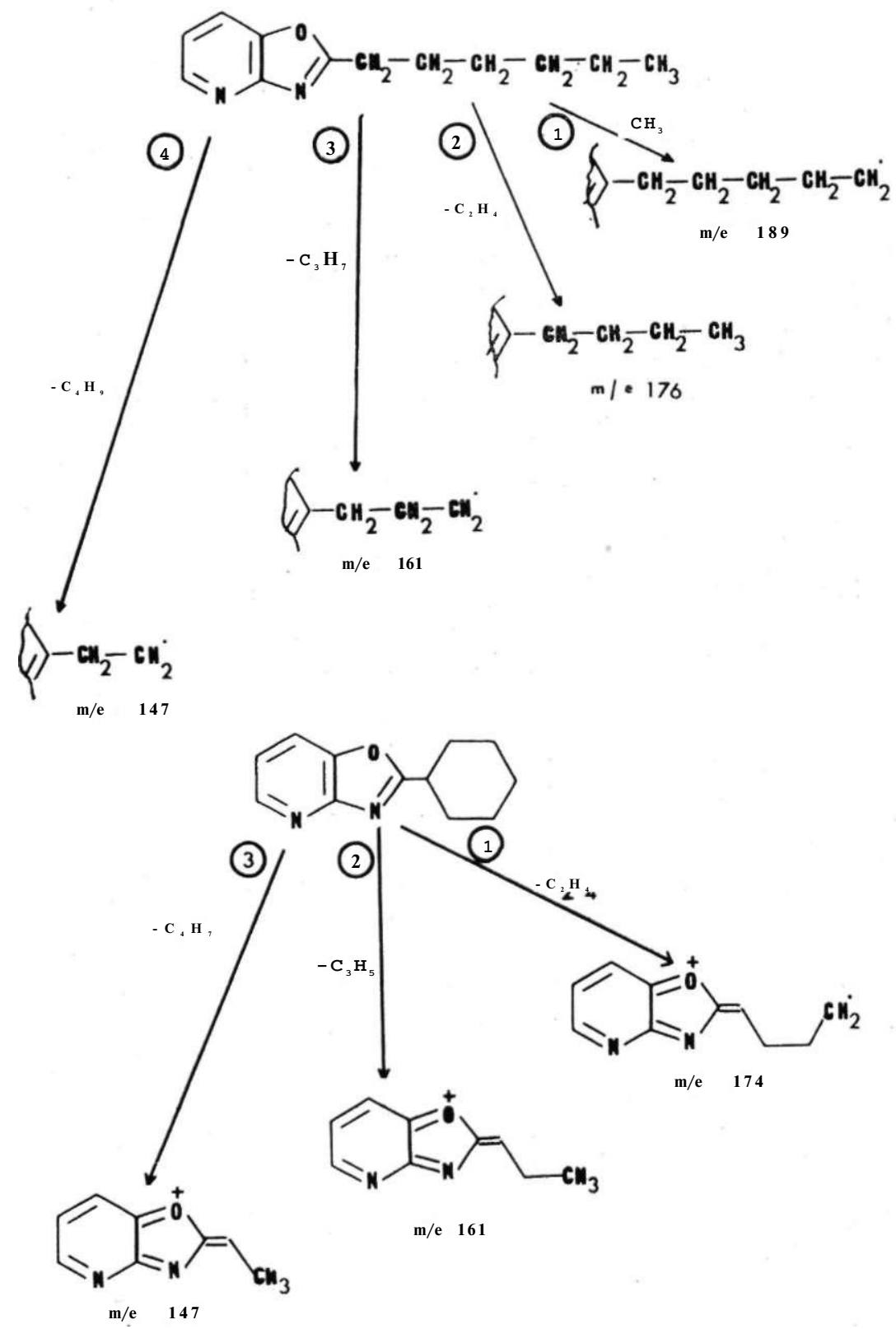


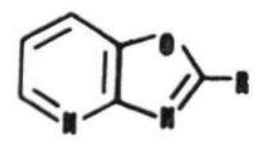

Tablo 1 Sentezi yapılan oksazolopiridin türevleri ve ergime veya kaynama dereceleri.

\begin{tabular}{|c|c|c|c|c|}
\hline $\begin{array}{l}T \text { ü } r \text {. } \\
\text { No }\end{array}$ & $R$ & E. n. & $\mathrm{K} \cdot \mathrm{n}$. & $\begin{array}{l}\text { E.n. } \\
(\text { Lit.) }\end{array}$ \\
\hline I & $\mathrm{CH}_{3}$ & $71.0^{\circ} \mathrm{C}$ & & $74^{\circ} \mathrm{C}(11)$ \\
\hline 11 & $\mathrm{C}_{2} \mathrm{H}_{5}$ & $52.3^{\circ} \mathrm{C}$ & & $53^{\circ} \mathrm{C}(3)$ \\
\hline I I I & - $\mathrm{n}-\mathrm{C}_{3} \mathrm{H}_{7}$ & $60.5^{\circ} \mathrm{C}$ & & 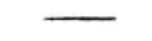 \\
\hline IV & - $\quad \mathrm{i}-\mathrm{C}_{3} \mathrm{H}_{7}$ & $\longrightarrow$ & $260^{\circ} \mathrm{C}$ & - \\
\hline V & - $\quad \mathrm{n}-\mathrm{C}_{4} \mathrm{H}_{9}$ & $25.0^{\circ} \mathrm{C}$ & & \\
\hline VI & - i $-\mathrm{C}_{4} \mathrm{H}_{9}$ & - & $270^{\circ} \mathrm{C}$ & \\
\hline V I I & - $\quad \mathrm{n}-\mathrm{C}_{5} \mathrm{H}_{11}$ & $37.0^{\circ} \mathrm{C}$ & 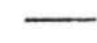 & \\
\hline VIII & - $\quad \mathrm{n}-\mathrm{C}_{6} \mathrm{H}_{13}$ & $38.5^{\circ} \mathrm{C}$ & & \\
\hline$I X$ & & & $285^{\circ} \mathrm{C}$ & \\
\hline$x$ & & $42.1^{\circ} \mathrm{C}$ & & \\
\hline$x \mid$ & & $58.9^{\circ} \mathrm{C}$ & & \\
\hline$x \mid 1$ & & $90.6^{\circ} \mathrm{C}$ & & $95^{\circ} \mathrm{C}(3)$ \\
\hline
\end{tabular}

* illk kez bu çalışmada sentezlenmiştir. 
NMR Spektrumu: $\delta$ ppm. 2.57 (metil grubunun protonlar, $3 \mathrm{H}$, t), 7.15 (6. konumdaki proton, $1 \mathrm{H}, \mathrm{dd}), 7.07$ (7. konumdaki proton, $1 \mathrm{H}, \mathrm{d}), 8.43$ (5. konumdaki proton, $1 \mathrm{H}, \mathrm{d})$.

Kütle Spektrumu: $M+134$ Moleküler iyon (\% 100), m/e 93 $(\mathrm{M}+-41)$ oksazol halkasının yanlışı (\% 96.66), m/e 65 (m/e 93-28) CO kopuşu (\% 50).

\section{2) 2-Etiloksazolo (4,5-b) piridin (Bileşik No: II)}

Genel yöntem uygulanan sentezde, $2 \mathrm{~g}(0.018$ mol) 2-amino-3hidroksipiridin, $5.8 \mathrm{~g}(0.045 \mathrm{~mol})$ propiyonik anhidrit kullanıld $1.150^{\circ} \mathrm{C}$ de yürütülen reaksiyon sonucunda $0.583 \mathrm{~g}$ kristal bileşik elde edildi. (Verim: \% 21.67)

NMR Spektrumu: $\delta$ pp. 1.28 (etil grubunun metil protonlar 1 , $3 \mathrm{H}, \mathrm{t}$ ), 2.86 (etil grubunun metilen protonlar $1,2 \mathrm{H}, \mathrm{k}$ ), 7.22 (6. konumdaki proton, $1 \mathrm{H}, \mathrm{dd}), 7.72$ (7. konumdaki proton, $1 \mathrm{H}, \mathrm{d}), 8.48$ (5. konumdaki proton, $1 \mathrm{H}, \mathrm{d}$ ).

Kütle Spektrumu: $M+148$ Moleküleriyon (\% 81.91), m/e 147 $(\mathrm{M}+-1)$ hidrojen kopuşu (\% 58.51), m/e 133 ( $\mathrm{M}+-15) \quad \mathrm{CH}_{3}$ kopuşu (\% 14.89), m/e 93 ( $\mathrm{M}+-55)$ oksazol halkasının yanlışı (\% 100), $\mathrm{m} / \mathrm{e}$ 65 (m/e 93-28) CO kopuşu (\% 45.74).

\section{3) 2 -n-Propiloksazolo (4,5-b) piridin (Bileşik No: III)}

Genel yöntem uygulanan sentezde, $5 \mathrm{~g}$ (0.045 mol) 2-amino-3hidroksipiridin, $11.9 \mathrm{~g}(0.135 \mathrm{~mol})$ n-butirik asit kullanıld $1.160^{\circ} \mathrm{C}$ de yürütülen reaksiyon sonucunda $2.1 \mathrm{~g}$ kristal bileşik elde edildi. (Verim: \% 28.53).

NMR Spektrumu: $\delta$ ppm. 1.07 (n-propil grubunun metil protonlar1, 3H, t), 1.95 (n-propil grubunun metilen protonlar1, 2 H, m) 2.97 (n-propil grubunun 1 numaral1 metilen protonlar $1,2 \mathrm{H}, \mathrm{t}), 7.22$ (6. konumdaki proton, $1 \mathrm{H}, \mathrm{dd}), 7.80$ (7. konumdaki proton, $1 \mathrm{H}, \mathrm{d}), 8.50$ (5. konumdaki proton, $1 \mathrm{H}, \mathrm{d}$ ).

Kütle Spektrumu: $M+162$ Moleküleriyon $(\%$ 14.66), m/e 148 $(\mathrm{M}+-14) \quad \mathrm{CH}_{2}$ kopuşu (\% 18.66), m/e $147 \quad(\mathrm{M}+-15) \quad \mathrm{CH}_{3}$ kopuşu (\% $32), \mathrm{m} / \mathrm{e} 134(\mathrm{M}+-28) \quad \mathrm{C}_{2} \mathrm{H}_{4}$ klopuşu (\% 100), m/e 93 (M+-69) oksazol halkasının yarılışı (\% 64), m/e 65 (m/e 93-98) CO kopuşu (\% 40). 


\section{4) 2 -İzopropiloksazolo (4,5-b) piridin (Bileşik No: IV)}

Genel yöntem uygulanan sentezde, $5 \mathrm{~g}$ (0.045 mol) 2-amino-3hidroksipiridin, $11.9 \mathrm{~g}(0.135 \mathrm{~mol})$ izobutirik asit kullanıld $1.140{ }^{\circ} \mathrm{C}$ de yürütülen reaksiyon sonucunda 2.253 g sıvı bileşik elde edildi. (Verim: \% 30. 61)

NMR Spektrumu: $\delta$ ppm. 1.48 (izopropil grubunun metil protonlar1 $6 \mathrm{H}, \mathrm{d}$ ), 3.30 (izopropil grubunun metin protonu, $1 \mathrm{H}, \mathrm{m}$ ), 7.30 (6. konumdaki proton, $1 \mathrm{H}, \mathrm{dd}$ ), 7.95 (7. konumdaki proton, $1 \mathrm{H}$, d), 8.60 (5. konumdaki proton, $1 \mathrm{H}, \mathrm{d})$.

Kütle Spektrumu: $\mathbf{M}^{+} 162$ Moleküler iyon (\% 66.25), m/e 147 ( M M 5 ) $\quad \mathrm{CH}_{3}$ kopuşu (\% 100), m/e $134(\mathrm{M}+-28) \quad \mathrm{C}_{2} \mathrm{H}_{4}$ kopuşu (\% 25), m/e $93\left(\mathrm{M}^{+}-69\right)$ oksazol halkasının yanlışı (\% 80), m/e 65 (m/e 93-28) CO kopuşu (\% 41.25).

\section{5) 2-n- Butiloksazolo (4,5-b) piridin (Bileşik No: V)}

Genel yöntem uygulanan sentezde, 5 g (0.045 mol) 2-amino-3hidroksipiridin, $13.8 \mathrm{~g}(0.135 \mathrm{~mol})$ n-valerik asit kullanıld $1.160^{\circ} \mathrm{C}$ de yürütülen reaksiyon sonucunda $3.38 \mathrm{~g}$ kristal bileşik elde edildi. (Verim: \% 42.25)

NMR Spektrumu: $\delta$ ppm. 1.03 (n-butil grubunun metil protonlar1, $3 \mathrm{H}, \mathrm{t}$ ), 1.52 (n-butil grubunun 3 numaral1 metilen protonlar $2 \mathrm{H}, \mathrm{m}$ ), 1.96 (n-butil grubunun 2 numaral1 metilen protonlar, $2 \mathrm{H}$, m), 3.05 (n-butil grubunun 1 numaral1 metilen protonlar $1,2 \mathrm{H}, \mathrm{t}$ ), 7.33 (6. konumdaki proton, $1 \mathrm{H}, \mathrm{dd}$ ), 7.92 (7. konumdaki proton, $1 \mathrm{H}$, $1 \mathrm{H}, \mathrm{d}), 8.65$ (5. konumdaki proton, $1 \mathrm{H}, \mathrm{d})$.

Kütle Spektrumu: M+ 176 Moleküler iyon (\% 3.36), m/e 175 $(\mathrm{M}+-1)$ hidrojen kopuşu (\% 2.85), m/e $161 \quad(\mathrm{M}+-15) \mathrm{CH}_{3}$ kopuşu '(\% 3.69), m/e $148(\mathrm{M}+-28) \quad \mathrm{C}_{2} \mathrm{H}_{4}$ kopuşu (\% 20.43), m/e 134 (M+ -42) $\mathrm{C}_{3} \mathrm{H}_{6}$ kopuşu (\% 100), m/e 93 ( $\left.\mathrm{M}+-83\right)$ oksazol halkasının yanlış1 (\% 47.31), m/e 65 (m/e 93-28) CO kopuşu (\% 33.33).

\section{6) 2 -tzobutiloksazolo (4,5-b) piridin (Bileşik No: VI)}

Genel yöntem uygulanan sentezde, $5 \mathrm{~g}$ (0.045 mol) 2-amino-3hidroksipiridin, $13.8 \mathrm{~g}(0.135 \mathrm{~mol})$ izovalerik asit kullanıld $1.150^{\circ} \mathrm{C}$ de yürütülen sentez sonucunda $3.6 \mathrm{~g}$ sıvı bileşik elde edildi. (Verim: $\%$ 45). 
NMR Spektrumu: $\delta$ ppm. 1.09 (izobutil grubunun metil protonlar1, 6H, d), 2.35 (izobutil grubunun metin protonu, $1 \mathrm{H}, \mathrm{m}$ ) 2.90 (izobutil grubunun metilen protonu, $2 \mathrm{H}$, d), 7.28 (6. konumdaki proton, $1 \mathrm{H}, \mathrm{dd}), 7.87$ (7. konumdaki proton, $1 \mathrm{H}, \mathrm{d}), 8.57$ (5. konumdaki proton, $1 \mathrm{H}, \mathrm{d})$.

Kütle Spektrumu: $\mathrm{M}+176$ Moleküler iyon (\% 24.57), m/e 161 ( $\mathrm{M}+-15) \quad \mathrm{CH}_{3}$ kopuşu (\% 52.54), m/e 147 ( $\left.\mathrm{M}+-29\right) \quad \mathrm{C}_{2} \mathrm{H}_{5}$ kopuşu (\% 18.64), m/e $134(\mathrm{M}+-42) \mathrm{C}_{3} \mathrm{H}_{6}$ kopuşu (\% 100), m/e 93 ( $\left.\mathrm{M}+-83\right)$ oksazol halkasının yanlışı (\% 72.88), m/e 65 (m/e 93-28) CO kopuşu (\% 45.76).

\section{7) 2-n- Pentiloksazolo (4,5-b) piridin (Bileşik No: VII)}

Genel yöntem uygulanan sentezde, $1.5 \mathrm{~g}(0.013 \mathrm{~mol})$ 2-amino-3hidroksipiridin, $4.53 \mathrm{~g}(0.039 \mathrm{~mol})$ heksanoik asit kullanıld $1.170^{\circ} \mathrm{C}$ de yürütülen reaksiyon sonucunda $1.26 \mathrm{~g}$ kristal bileşik elde edildi. (Verim: \% 48.64)

NMR Spektrumu: $\delta$ ppm. 0.97 (n-pentil grubunun metil protonlar $1,3 \mathrm{H}, \mathrm{t}$ ), 1.43 (n-pentil grubunun 3 ve 4 numaralı metilen protonlar1, 4H, m), 1.95 (n-pentil grubunun 2 numaralı metilen protonlar1, 2H, m), 3.01 (n-pentil grubunun 1 numaral1 metilen protonlar1, $2 \mathrm{H}, \mathrm{t}), 7.25$ (6. konumdaki proton, $1 \mathrm{H}, \mathrm{dd}), 7.85$ (7. konumdaki proton, $1 \mathrm{H}, \mathrm{d}), 8.58$ (5. konumdaki proton, $1 \mathrm{H}, \mathrm{d})$.

Kütle Spektrumu: M+ 190 Moleküler iyon (\% 4.22), m/e 176 $(\mathrm{M}+-14) \quad \mathrm{CH}_{2}$ kopuşu (\% 7.04), m/e $162(\mathrm{M}+-28) \quad \mathrm{C}_{2} \mathrm{H}_{4}$ kopuşu (\% 16.90), m/e $147(\mathrm{M}+-43) \quad \mathrm{C}_{3} \mathrm{H}_{7}$ kopuşu (\% 20.42), m/e 134 ( $\mathrm{M}+-$ 56) $\mathrm{C}_{4} \mathrm{H}_{8}$ kopuşu (\% 100), m/e 93 ( $\left.\mathrm{M}+-97\right)$ oksazol halkasının yanlış1 (\% 28.16), m/e 65 (m/e 93-28) CO kopuşu (\% 21.12).

8) 2-n- Hekzilosakzolo (4,5-b) piridin (Bileşik No: VIII)

Genel yöntem uygulanan sentezde, $1.5 \mathrm{~g}(0.013 \mathrm{~mol})$ 2-amino -3-hidroksipiridin, 5g $(0.039 \mathrm{~mol}) \mathrm{n}$-hepatonik asit kullan $1 \mathrm{ld} 1.170^{\circ} \mathrm{C}$ de yürütülen reaksiyon sonucunda $1.62 \mathrm{~g}$ kristal bileşik elde edildi. (Verim: \% 58.27)

NMR Spektrumu: $\delta$ ppm. 0.90 (n-hekzil grubunun metil protonlar $1,3 \mathrm{H}, \mathrm{t}$ ), 1.35 (n-hekzil grubunun 3,4 ve 5 numaralı metilen 
protonlar $1,6 \mathrm{H}, \mathrm{m}), 1.90$ (n-hekzil grubunun 2 numaralı metilen protonlar $1,2 \mathrm{H}, \mathrm{m}), 2.96$ (n-hekzil grubunun 1 numaral1 metilen protonlar1, $2 \mathrm{H}, \mathrm{t}$ ), 7.22 (6. konumdaki proton, $1 \mathrm{H}, \mathrm{dd}), 7.75$ (7. konumdaki proton, $1 \mathrm{H}, \mathrm{d}), 8.50$ (5. konumdaki proton, $1 \mathrm{H}, \mathrm{d})$.

Kütle Spektrumu: $\mathbf{M}^{+} 204$ Moleküler iyon $(\% 8.40), \mathrm{m} / \mathrm{e} 189$ $(\mathrm{M}+-15) \quad \mathrm{CH}_{3}$ kopuşu $\left(\%\right.$ 11.76), m/e $176 \quad(\mathrm{M}+-28) \quad \mathrm{C}_{2} \mathrm{H}_{4}$ kopuşu $\left(\begin{array}{ll}\% & 14.76\end{array}\right), \mathrm{m} / \mathrm{e} 161(\mathrm{M}+-43) \quad \mathrm{c}_{3} \mathrm{H}_{7}$ kopuşu $(\%$ 13.86), m/e 147 ( $\mathrm{M}+-$ 57) $\mathrm{C}_{4} \mathrm{H}_{9}$ kopuşu $\left(\%\right.$ 36.13), m/e $134(\mathrm{M}+-70) \quad \mathrm{C}_{5} \mathrm{H}_{10}$ kopuşu (\% $100), \mathrm{m} / \mathrm{e} 93(\mathrm{M}+-111)$ oksazol halkasının yanlış1 (\% 25.21), $\mathrm{m} / \mathrm{e}$ 65 (m/e 93-28) CO kopuşu (\% 21.84).

\section{9) 2- Siklopropiloksazolo (4,5-b) piridin (Bileşik No: IX)}

Genel yöntem uygulanan sentezde, 5 g (0.045 mol) 2-amino-3hidroksipiridin, $11.6 \mathrm{~g}(0.135 \mathrm{~mol})$ siklopropil karboksilik asit kullanıldı. $100{ }^{\circ} \mathrm{C}$ de yürütülen reaksiyon sonucunda 2.2 g sıvı bileşik elde edildi (Verim: \% 30.26)

NMR Spektrumu: $\delta$ ppm. 1.05 (siklopropil halkasının metilen protonlar $1,4 \mathrm{H}, \mathrm{m}$ ), 2.05 (siklopropil halkasinın metin protonu, $1 \mathrm{H}$, m), 7.24 (6. konumdaki proton, İH, dd), 7.80 (7. konumdaki proton, $1 \mathrm{H}, \mathrm{d}), 8.55$ (5. konumdaki proton, $1 \mathrm{H}, \mathrm{d})$.

Kütle Spektrumu: $M+160$ moleküler iyon (\% 100), m/e 159 $(\mathrm{M}+-1)$ hidrojen kopuşu (\% 41.13), m/e $134(\mathrm{M}+-26) \mathrm{C}_{2} \mathrm{H}_{2}$ kopuşu (\% 21.27), m/e $93(\mathrm{M}+-67)$ oksazol halkasının yanlış1 (\% 83.68), $\mathrm{m} / \mathrm{e} 65(\mathrm{~m} / \mathrm{e}$ 92-38) CO kopuşu (\% 60.28)

\section{0) 2- Siklobutiloksazolo (4,5-b) piridin (Bileşik No: X)}

Genel yöntem uygulanan sentezde, $5 \mathrm{~g}$ (0.045 mol) 2-amino-3hidroksipiridin, $13.5 \mathrm{~g}(0.135 \mathrm{~mol})$ siklobutan karboksilik asit kullanıld1. $150^{\circ} \mathrm{C}$ de yürütülen reaksiyon sonucunda $3.86 \mathrm{~g}$ kristal bileşik elde edildi. (Verim: \% 48.86)

NMR Spektrumu: $\delta$ ppm. 1.90-2.85 (siklobutil halkasının metilen protonları, $6 \mathrm{H}, \mathrm{m}$ ), 3.67-4.07 (siklobutil halkasinın metin proto$\mathrm{nu}, 1 \mathrm{H}, \mathrm{m}), 7.29$ (6. konumdaki proton, $1 \mathrm{H}, \mathrm{dd}), 7.85$ (7. konumdaki proton, $1 \mathrm{H}, \mathrm{d}), 8.60$ (5. konumdaki proton, $1 \mathrm{H}, \mathrm{d})$. 
Kütle Spektrumu: $\mathrm{M}+174$ Moleküler iyon (\% 13.47), m/e 173 $(\mathrm{M}+-1)$ hidrojen kopuşu (\% 4.96), m/e $146(\mathrm{M}+-28) \mathrm{C}_{2} \mathrm{H}_{4}$ kopuşu (\% 100), m/e $93(\mathrm{M}+-81)$ oksazol halkasının yanlışı (\% 47.51), m/e 65 (m/e 93-29) CO kopuşu (\% 24.11)

\section{1) 2-Siklopentiloksazolo (4,5-b) piridin (Bileşik No: XI)}

Genel yöntem uygulanan sentezde, $5 \mathrm{~g}$ (0.045 mol) 2-amino-3hidroksipiridin, $15.5 \mathrm{~g}(0.135 \mathrm{~mol})$ siklopentan karboksilik asit kullanıld $1.150{ }^{\circ} \mathrm{C}$ de yürütülen reaksiyon sonucunda $3.65 \mathrm{~g}$ kristal bileşik elde edildi. (Verim: \% 42.74)

NMR Spektrumu: $\delta$ p pm. 1.55-2.45 (siklopentan halkasının metilen protonlar $1,8 \mathrm{H}, \mathrm{m}$ ), 3.25-3.65 (siklopentan halkasının metin protonu, $1 \mathrm{H}, \mathrm{m}), 7.27(6$. konumdaki proton, $1 \mathrm{H}, \mathrm{dd}), 7.83$ (7. konumdaki proton, $1 \mathrm{H}, \mathrm{d}), 8.58$ (5. konumdaki proton, $1 \mathrm{H}, \mathrm{d})$.

Kütle Spektrumu: M+ 188 Moleküler iyon (\% 13.47), m/e 187 $(\mathrm{M}+-1)$ hidrojen kopuşu (\% 11.34), m/e $160(\mathrm{M}+-28) \mathrm{C}_{2} \mathrm{H}_{4}$ kopuşu (\% 18.43), m/e 147 ( $\mathrm{M}+-41) \quad \mathrm{C}_{3} \mathrm{H}_{5}$ kopuşu (\% 100), m/e 93 ( $\mathrm{M}+$ 95) oksazol halkasının yanlışı (\% 29.78), m/e 65 (m/e 93-28) CO kopuşu (\% 24.82).

12) 2-Siklohekziloksazolo (4,5-b) piridin (Bileşik No: XII)

Genel yöntem uygulanan sentezde, 5 g (0.045 mol) 2-amino-3Lidroksipiridin, $17.4 \mathrm{~g}(0.135 \mathrm{~mol})$ siklohekzan karboksilik asit kullanıld $1.170^{\circ} \mathrm{C}$ de yürütülen reaksiyon sonucunda $4.1 \mathrm{~g}$ kristal bileşik elde edildi (Verim: \% 44.66)

NMR Spektrumu: $\delta$ p pm. 1.03-2.33 (siklohekzan halkasının metilen protonları, $1 \mathrm{OH}, \mathrm{m}$ ), 2.78-3.13 (siklohekzan halkasının metin protonu, $1 \mathrm{H}, \mathrm{m}), 7.18$ (6. konumdaki proton, $1 \mathrm{H}, \mathrm{dd}), 7.27$ (7. konumdaki proton, $1 \mathrm{H}, \mathrm{d}), 8.46$ (5. konumdaki proton, $1 \mathrm{H}, \mathrm{d})$.

Kütle Spektrumu: M+ 202 Moleküler iyon (\% 8.74), m/e 201 $(\mathrm{M}+-1)$ hidrojen kopuşu (\% 12.24), m/e $174(\mathrm{M}+-28) \mathrm{C}_{2} \mathrm{H}_{4}$ kopuşu (\% 10.49), m/c 161 ( M+-41) $\mathrm{C}_{3} \mathrm{H}_{5}$ kopuşu (\% 15.74), m/e 147 (M+55) $\mathrm{C}_{4} \mathrm{H}_{7}$ kopuşu (\% 44.89), m/e 134 ( $\left.\mathrm{M}+-68\right) \mathrm{C}_{5} \mathrm{H}_{8}$ kopuşu (\% 100), $\mathrm{m} / \mathrm{e} 93(\mathrm{M}+-109)$ oksazol halkasının yanlışı (\% 32.65), m/e 65 (m/e 93-28) CO kopuşu (\% 26.53). 


\section{SONUÇ ve TARTIŞMA}

Bileşiklerin sentezi, seçilen karboksilik asitler veya anhidritleri ile 2 amin.o-3-hidroksipiridinin polifosforik asitli ortamda 1sıtılması ile yapılmıştır. Ancak karboksilik asitlerden hareketle yapılan sentezlerde ortama su çekici ajan olarak polifosforik asitin yanı sıra fosfor pentaoksitin de kullanılması oksazolopiridin türevlerinin sentezinde ilk kez denenmiştir. Bu şekilde fosfor pentaoksit ilavesinin, sentez verimini artırdı̆̆ 1 gözlenmiştir.

Gerçekleştirilen yapı aydınlatmaları sonucunda, 9 tanesi ilk kez bu çalışmada hazırlanmış olan 12 adet 2-sübstitüeoksazolo (4,5-b) piridin türevi sentezlenmiştir.

\section{L İ T E R A T Ü R}

1. Fraser, J. and Tittensor, E., Oxazolopyridincs and Oxazoloquinolines, J. Chem. Soc, 4625, (1957)

2. Clark, R. L., Pessolano. A. A., Witzel, B., Lanza, T., Shen, T. Y., 2- (Substitutedphenyl) oxazolo (4,5-b) pyridines and 2- (Substituted-phenyl) oxalzolo (5,4-b) pyridines as Nonacidic Antienflammatory Agents, J. Med. Chem., 21, 1158 (1978)

3. Shen, T. Y., Clark, R. L., Pessolano, A. A., Witzel, B. Lanza, T., Antienflamma tory Oxazolo (4,5-b) pyridines, U. S. Pat., 4.038.396, 26 Jul 1977.

4. Shen, T. Y., Clark, R. L., Pessolano, A. A.., Witzel, B., Lanza, T., Oxazolo (4,5-b) pyridines, Can. Pat., 1.032.166, 30 May 1978.

5. Shen, T. Y., Clark, R. L., Pessolano, A. A., Witzel, B., Lanza, T., Oxazolopyridines and Thiazolopyridines, The Patent Office London, 1.421.619, 11 Jun 1973.

6. Shen, T. Y., Clark, R. L., Pessolano, A. A., Witzel, B., Lanza, T., New Synthetic Method for Derivatives of 1,4-Dihydropyrazincs, Ger. Pat., 2.527.321, 23 Dec. 1976.

7. Ruefenacht, K., Kristinsson, H., Derivate von Oxazolo (4,5-b) pyridin-2 (3H) on, Helv. Chim. Acta., 59, 1593 (1976)

8. Alt, K. O., Christen, E., and Weis, C. D., Trihalogenated Amino-pyridinols, J. Heterocycl. Chem., 12, (4), 775 (1975)

9. Philippe. J., 2- Aminooxazolo (4,5-b) pyridines, Ger., Pat., 2.239.311, 22 Feb 1973.

10. Ferlux-Chimie S. A., 2-Thiooxazolo (4,5-b) pyridines, Fr. Pat., 2. 190.426, 27 Jun 1972

11. Takahashi, T., and Yoneda, F., The Syntheses of Derivatives of 3-hydroxypridines, Pharm. Bull, 5, 350-3 (1957). Ref.: C. A., 52, 13723 (1958)

12. Hisano, T., Matsuoka, T., Ichikawa, M., Reaction of 3,5-Dibromopyridine N-oxide with Phenyl Isocyanate, Heterocycles, 2, (2) 163-6 (1974), Ref.: C. A., 80, 146063w (1974) 